\title{
Quantum Criticality in Dimerized Spin Ladders
}

\author{
Gennady Y. Chitov, Brandon W. Ramakko, and Mohamed Azzoud* \\ Department of Physics, Laurentian University, Ramsey Lake Road, Sudbury, ON, P3E 2C6, Canada
}

(Dated: August 4, 2021)

\begin{abstract}
We analyze a possibility of quantum criticality (gaplessness) in dimerized antiferromagnetic twoand three-leg spin- $\frac{1}{2}$ ladders. Contrary to earlier studies of these models, we examine different dimerization patterns in the ladder. We find that ladders with the columnar dimerization order have lower zero-temperature energies and they are always gapped. For the staggered dimerization order, we find the quantum critical lines, in agreement with earlier analyses. The bond meanfield theory we apply, demonstrates its quantitative accuracy and agrees with available numerical results. We conclude that unless some mechanism for locking dimerization into the energetically less favorable staggered configuration is provided, the dimerized ladders do not order into the phase where the quantum criticality occurs.
\end{abstract}

PACS numbers: 75.10.Jm, 75.10.-b, 75.10.Pq, 73.43.Nq, 64.60.-i

\section{INTRODUCTION}

There has been a lot of interest in spin ladders for more than a decade by now, mainly due to their very intriguing critical properties. One of the most peculiar ones is that the existence of a gap (i.e. mass) depends on the number of legs. The spin excitations in a $m$-leg spin ladder are gapped if $m$ is even, and the system is gapless (quantum critical) when the number of legs $m$ is odd. For reviews see Ref.[1,2]. The even- $m$-leg ladders provide a very interesting example of systems where the gap (mass) generation is not accompanied by a long-range order or apparent symmetry breaking. Such systems, known as spin liquids, are under enormous scrutiny. They are notoriously difficult to realize in dimension more than one, and are believed to be relevant to the physics of high- $T_{c}$ superconductors. $\stackrel{3}{-}$ Broadly speaking, spin ladders are interesting for studying gap (mass) generation: needless to say that this a very deep question in all physics.

The particular question we address in this study is a possibility of "restored quantum criticality" (or gaplessness) in a system (ladder) built from gapped blocks (dimerized chains) $4,5,6,7,8,9,10,11$ Let us explain the issue taking a dimerized two-leg ladder as an example. ${ }^{12}$ It is well known that a single Heisenberg spin- $\frac{1}{2}$ chain with alternating spin exchange (a.k.a. the dimerized Heisenberg chain) is gapped. $\stackrel{2}{2}$ When two chains are coupled into a ladder, the system is gapped even without dimerization. However, quite remarkably and counterintuitively, the dimerized two-leg ladder can be gapless, as was conjectured in Ref. [4]. Subsequent numerical work $k^{5,6,7}, 9$ confirmed the critical (gapless) line proposed by MartinDelgado et $a l^{4}$ Similarly, the originally conjectured critical line in a dimerized three-leg ladder ${ }^{4}$ was confirmed in the very recent DMRG calculations. ${ }^{10,11}$

We need to stress one very important point: in the earlier work ${ }^{5,6,7,8,9,10,11}$ on the intrinsically dimerized ladders, the intra-chain dimerization as well as the (staggered) dimerization ordering pattern of the whole ladder (cf. Figs. 16 below) are taken for granted, i.e., as the model built-in assumptions. In the absence of a physical mechanism which would lock a ladder with dimerized chains into a particular dimerization order, it is natural and physically more reasonable to consider various possible ordering patterns on the same footing. It means that the possibility of quantum criticality (gaplessness) has to be addressed in this broader setting. A strong motivation comes from a recent work on the three-leg ladder coupled to phonons. ${ }^{13}$ It turns out that the phonon-induced intrachain dimerizaton, appearing in this ladder at the spin-Peierls transition, occurs into the columnar dimerization order (cf. Fig. 6] below). The latter, and not the staggered order, minimizes the spin-phonon Hamiltonian. The columnar dimerized three-leg ladder is always gapped.

So, we begin from the study of the dimerized two-leg ladder in Sec. II. From analyses of the limits where the behavior of this ladder is known exactly, we conjecture that the gapped columnar dimerized phase has lower energy. This is confirmed by the subsequent treatment within the bond mean-field theory $14,15,16$ The latter approach, when applied for the case of staggered dimerization, demonstrates a good agreement with available numerical results, and also yields quite simple formulas for analytical treatments of the problem. A similar program with analogous conclusions is carried out for the three-leg ladder in Sec. III.

\section{TWO-LEG LADDER}

We consider a two-leg dimerized spin ladder given by the Hamiltonian:

$$
\begin{aligned}
H_{2 \mathrm{~L}} & =\sum_{\alpha=1,2} \sum_{n=1}^{N} J_{\alpha}(n) \mathbf{S}_{\alpha}(n) \cdot \mathbf{S}_{\alpha}(n+1) \\
& +J_{\perp} \sum_{n=1}^{N} \mathbf{S}_{1}(n) \cdot \mathbf{S}_{2}(n) .
\end{aligned}
$$


The $m$-leg ladder has $N$ rungs and $m \cdot N$ spins. We consider the situation when dimerization occurs along the chains $(\alpha=1,2)$ only, while the coupling $J_{\perp}$ is constant on each rung. One needs to consider two possible dimerization patterns: alternated, when staggering occurs in both directions

$$
J_{\alpha}(n)=J\left[1+(-1)^{n+\alpha} \delta\right],
$$

and columnar, when

$$
J_{\alpha}(n)=J\left[1+(-1)^{n} \delta\right]
$$

The two patterns are shown in Fig. 1]

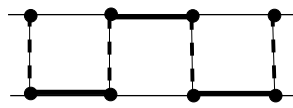

(a)

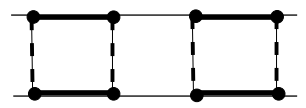

(b)
FIG. 1: Dimerized two-leg ladder. Bold/thin/dashed lines represent the stronger/weaker chain coupling $J(1 \pm \delta)$ and rung coupling $J_{\perp}$, respectively. Dimerization patterns: (a) staggered; (b)- columnar.

\section{A. Analytical Limits}

Let us discuss the points on the $\left(\delta, J_{\perp}\right)$-plane where the properties of the model (1) are known.

$\delta=0, J_{\perp}=0-$ two decoupled critical (gapless) Heisenberg chains.

$\delta=0, J_{\perp}>0-$ uniform gapped ladder.

$\delta=1$ - complete dimerization. In this limit the model reduces to:

(a) the snake-like dimerized Heisenberg chain of $2 N$ spins in case of the alternated staggered pattern (Fig. 2a)

(b) a set of $N / 2$ decoupled four-spin plaquettes in case of the columnar dimerization pattern (Fig. 2b) For a four-

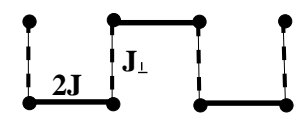

(a)

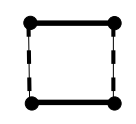

(b)

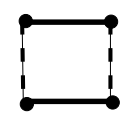

FIG. 2: Completely dimerized ladder, $\delta=1$. (a): Alternated staggering reduces the model (1) to a snake-like dimerized Heisenberg chain of $2 N$ spins; (b): Columnar order degenerates into a set of $N / 2$ decoupled plaquettes.

spin plaquette where its nearest-neighbor spins interact via the Heisenberg couplings $J_{1}$ and $J_{2}$, the spectrum is known. ${ }^{17}$ The ground-state singlet has the energy

$$
\mathcal{E}_{\square}=-\frac{J_{1}+J_{2}}{2}-\sqrt{J_{1}^{2}+J_{2}^{2}-J_{1} J_{2}},
$$

and is separated by a gap from the closest triplet state with with the energy $\mathcal{E}_{t}=-\frac{J_{1}+J_{2}}{2}$.
As was first noted in Ref. 4, at the critical point $2 J=J_{\perp} \equiv J_{c}$ the snake-like chain (Fig. 2a) becomes just a uniform (gapless) Heisenberg model. Its groundstate energy is

$$
E_{\mathrm{HC}}=-2\left(\log 2-\frac{1}{4}\right) J_{c} N \approx-0.88 J_{c} N
$$

The set of $N / 2$ plaquettes (4) has a lower ground-state energy

$$
E_{\square}=-J_{c} N
$$

The simple results (516) question the very existence of the critical line predicted $\mathrm{in}^{4}$. That line was conjectured essentially from a continuity argument and analysis of the model in the two solvable (integrable) points $\delta=0, J_{\perp}=0$ and $\delta=1, J_{\perp}=2 J$. The latter was assumed to be a critical (gapless) point, since the alternated staggering pattern (thus the snake-like chain in the limit $\delta=1$ ) was assumed to be a ground state. The subsequent analyses addressed the issue of the critical line (within the alternated staggering pattern) more quantitatively, $, 5,6,7,8,9$ while, to the best of our knowledge, comparisons with the energy of the columnar pattern were not done. As one can see from (56), the gapped plaquettes have lower energy than the critical (gapless) snake-like Heisenberg chain. Note that the decoupled plaquettes at $\delta=1$ evolve smoothly from the columnar dimerization pattern at $\delta<1$.

Let us now look at the line $\delta=1, J_{\perp} \neq 2 J$. It is convenient to parameterize couplings as

$$
\begin{aligned}
2 J & \equiv J_{\circ}\left(1+\delta_{\text {eff }}\right), \\
J_{\perp} & \equiv J_{\circ}\left(1-\delta_{\text {eff }}\right) .
\end{aligned}
$$

The plaquette pattern results in the ground-state energy

$$
E_{\square}=-\frac{1}{2} J_{\circ} N\left(1+\sqrt{1+3 \delta_{\text {eff }}^{2}}\right) .
$$

The energy minimum of the snake-like configuration is given by the ground-state energy of the dimerized Heisenberg chain. The latter is known to be equivalent to the massive sine-Gordon (integrable) model, perturbed by the marginal (logarithmic) corrections $\stackrel{18,19}{ }$ A leading order ansatz reads as

$$
E_{\mathrm{DHC}} \approx-2 J_{\circ} N\left(\log 2-\frac{1}{4}+a_{\circ} \frac{\delta_{\mathrm{eff}}^{4 / 3}}{\ln \frac{\delta_{\circ}}{\delta_{\mathrm{eff}}}}\right),
$$

where from the recent numerical calculations ${ }^{20} a_{\circ} \approx 2.2$ and $\delta_{\circ} \approx 110$. To get a more accurate analytical expression for $E_{\mathrm{DHC}}$ is an involved problem (for a recent analysis and more references, see $\left.{ }^{21}\right)$. However numerically, the ansatz (10) or even the unperturbed sine-Gordon formula 21

$$
E_{\mathrm{SG}} \approx-2 J_{\circ} N\left(\log 2-\frac{1}{4}+0.2728 \delta_{\mathrm{eff}}^{4 / 3}\right)
$$

both work quite well. A direct inspection of Eqs. (9]10]11) (e.g., plots) clearly shows that the plaquettes again provide the lower energy state on the line $\delta=1$ near the point $J_{\perp}=2 J \Longleftrightarrow \delta_{\text {eff }}=0.22$ 


\section{B. Mean-Field Equations}

Away from integrable points we need to resort to approximations. We will treat the dimerized ladders in the framework of the bond mean-field theory. ${ }^{14,15}$ This approach for the case of dimerized three-leg ladder is described in detail in 13 , and the case of two legs is essentially a simplified version of the former. First, the spin Hamiltonian is mapped onto an interacting fermion problem via a $2 \mathrm{D}$ version of the Jordan-Wigner transformation, proposed by one us. 14 Then, the phase differences due to hopping of the fermions around any given elementary plaquette is approximated by $\pi$. The quartic fermionic terms $c_{i,}^{\dagger} c_{i, \alpha} c_{i+1, \alpha}^{\dagger} c_{i+1, \alpha}$ are treated within the Hartree-Fock approximation, i.e., are decoupled using the single-particle (bond) parameters. The latter are defined as

$$
Q_{+}=\left\langle c_{2 i, \alpha} c_{2 i+1, \alpha}^{\dagger}\right\rangle, Q_{-}=\left\langle c_{2 i+1, \alpha} c_{2 i+2, \alpha}^{\dagger}\right\rangle,
$$

with $\alpha=1,2$. A single bond parameter suffices in the rung direction: $P=\left\langle c_{2 i, \alpha} c_{2 i, \alpha+1}^{\dagger}\right\rangle$. Fourier transforming along the chain direction and using the Nambu formalism, the mean-field theory results in the single-particle effective Hamiltonian

$$
H^{\mathrm{s} / \mathrm{c}}=\sum_{k} \Psi_{k}^{\dagger} \mathcal{H}^{(\mathrm{s} / \mathrm{c})} \Psi_{k}+N C_{2}
$$

where the Hamiltonian density $\mathcal{H}^{\mathrm{s} / \mathrm{c}}$ is a $4 \times 4$ matrix and the Nambu spinor $\Psi_{k}^{\dagger} \equiv\left(\begin{array}{llll}c_{1 k}^{A \dagger} & c_{1 k}^{B \dagger} & c_{2 k}^{A \dagger} & c_{2 k}^{B \dagger}\end{array}\right)$. Here $c_{\alpha k}^{\sharp}$ is the Fourier transform of $c_{i \alpha}^{\sharp}$. To account for the dimerization (doubling of the lattice spacing), the lattice is subdivided into two sublattices A and B. The explicit form of the effective Hamiltonian (13) depends on the dimerization pattern (staggered or columnar) which is accounted for by an extra label $(\mathrm{s}, \mathrm{c})$ in the above equation, and

$$
C_{2}=J_{+}\left|Q_{+}\right|^{2}+J_{-}\left|Q_{-}\right|^{2}+J_{\perp}|P|^{2} .
$$

Diagonalization of $\mathcal{H}^{\mathrm{s} / \mathrm{c}}$ yields four energy eigenvalues $\pm E_{n}^{\mathrm{s}}(k), n=1,2$ :

$$
E_{1 / 2}^{\mathrm{s}}(k)=\frac{1}{2} \sqrt{W+J_{\perp 1}^{2} \mp 2 J_{\perp 1}\left(J_{1+}-J_{1-}\right) \cos k},
$$

for the staggered phase. We define

$$
\begin{aligned}
J_{1 \pm} & =J(1 \pm \delta)\left(1+2 Q_{ \pm}\right), J_{\perp 1}=J_{\perp}(1+2 P), \\
W & =J_{1+}^{2}+J_{1-}^{2}-2 J_{1+} J_{1-} \cos (2 k) .
\end{aligned}
$$

For the columnar pattern we obtain two doubly degenerate energy eigenvalues $\pm E^{\mathrm{c}}(k)$ :

$$
E^{\mathrm{c}}(k)=\frac{1}{2} \sqrt{W+J_{\perp 1}^{2}}
$$

The partition function of the single-particle Hamiltonian (13) can be found in a closed form, and the free energy per spin is

$$
F^{\sharp}=\frac{1}{m} C_{m}-\frac{\log 2}{\beta}-\frac{2 \mathfrak{g}}{m \pi \beta} \sum_{n} \int_{0}^{\frac{\pi}{2}} \log \cosh \frac{\beta E_{n}^{\sharp}(k)}{2} d k,
$$

where $\beta=1 / k_{B} T$ and $\mathfrak{g}$ is eigenvalue's degeneracy. We gave the above formula valid for $m$ legs. The mean-field equations are derived from minimization of the free energy (19) with respect to the mean-field parameters $Q_{ \pm}$ and $P$. These self-consistent integral equations are solved numerically.

\section{Analysis}

To start with, let us make the following observation: In the antiferomagnetic two-leg ladder (1) with intrinsically dimerized chains we cannot think of a particular mechanism to enforce a specific (i.e., staggered or columnar) dimerization pattern. So, having the dimerized chains, the ladder must admit the pattern which minimizes its free energy. Using Eq. (19), we compared the zero-temperature energies of two configurations in various parameter ranges. The characteristic results are given in Fig. 3. The columnar pattern corresponds the lower energies in all cases, so it is the thermodynamically stable state (phase). Now let us analyze the excitation

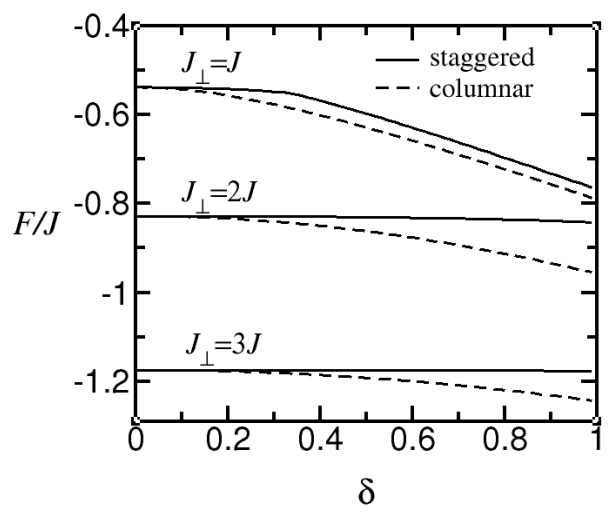

FIG. 3: Two-leg ladder: Energies of staggered and columnar dimerization configurations at $T=0$.

spectra of two phases. We will identify the minimal band gap of the eigenvalues (15) or (18) with the spin gap of the corresponding phase. To demonstrate that this is true, it suffices, e.g., to calculate the uniform spin susceptibility from the free energy (19) with an external magnetic field added.

We find that the columnar phase is always gapped with the gap $\Delta_{c}=E^{\mathrm{c}}(0)$ given by

$$
\Delta_{c}=J \sqrt{u^{2} \delta^{2}+\left(\frac{J_{\perp}}{2 J}\right)^{2} p^{2}}
$$




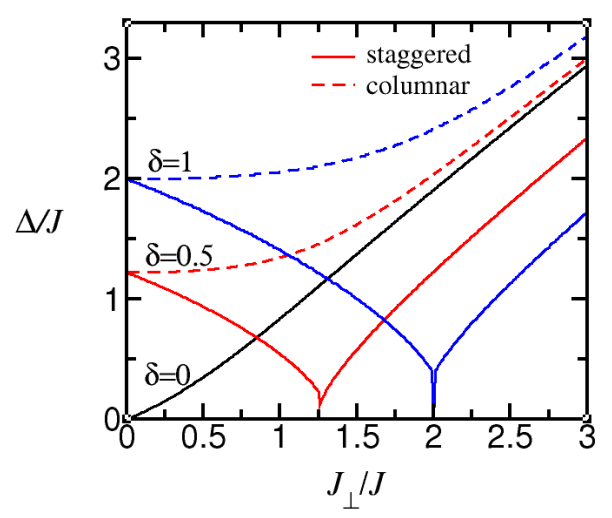

FIG. 4: Two-leg ladder: Gaps of the staggered and columnar phases.

where

$$
\begin{aligned}
& u=1+\left(Q_{+}+Q_{-}\right)+\frac{Q_{+}-Q_{-}}{\delta}, \\
& p=1+2 P .
\end{aligned}
$$

Qualitatively, the columnar phase is similar to the uniform two-leg ladder, i.e. gapped. The gap (20) persists in the limit of decoupled chains $J_{\perp} \rightarrow 0$, as it must be, and disappears only together with the vanishing chain dimerization $\delta \rightarrow 0$. If the latter limit is taken first, then the uniform ladder is gapped with $\Delta_{c}=J_{\perp} p / 2$.

The staggered phase is more interesting. Its gap $\Delta_{s}=$ $E_{1}^{\mathrm{s}}(0)$ reads as: $\underline{23}$

$$
\Delta_{s}=J\left|u \delta-\frac{J_{\perp}}{2 J} p\right| .
$$

It vanishes on a certain critical line, even if neither dimeritation $\delta$, nor rung coupling $J_{\perp}$ are zero. Thus, the mean-field theory confirms the earlier conjecture ${ }^{4}$, which has been so far corroborated only by numerical calculations $\stackrel{5,6,7,9}{ }$ With the bond parameters $Q_{ \pm}, P$ determined from the mean-field equations, we plot the gaps $\Delta_{c, s}$ given by Eqs. (20/23) as functions of coupling ratio $J_{\perp} / J$ for several dimerizations in Fig. 4. Note that the gaps $\Delta_{c}$ and $\Delta_{s}$ coincide at "the decoupled chains line" $J_{\perp} / J=0$, as well as in the absence of dimerization. The qualitative differences between the gaps in the columnar and staggered phases are clearly seen. The former is always non-zero if the chains are dimerized, and increases with the growth of $J_{\perp} / J$. The latter behaves non-monotonously, passing though a critical point. It is worth noting that the known analytical limits (discussed above) are recovered exactly by the mean-field equations. In particular, on the "decoupled" axis $J_{\perp}=0$, the meanfield parameter $Q_{ \pm}$whose physical meaning is the average of the dimerization operator $\mathbf{S}_{\alpha}(n) \cdot \mathbf{S}_{\alpha}(n+1)$, behaves almost as that in the dimerized $X Y$-chain, coinciding with the exact values of that quantity at the ends of the interval $\delta \in[0,1]$. For the dimerized $X Y$-chain

$$
Q_{ \pm}=\left\langle\mathbf{S}_{n} \cdot \mathbf{S}_{n+1}\right\rangle \equiv t \pm \delta \cdot \eta
$$

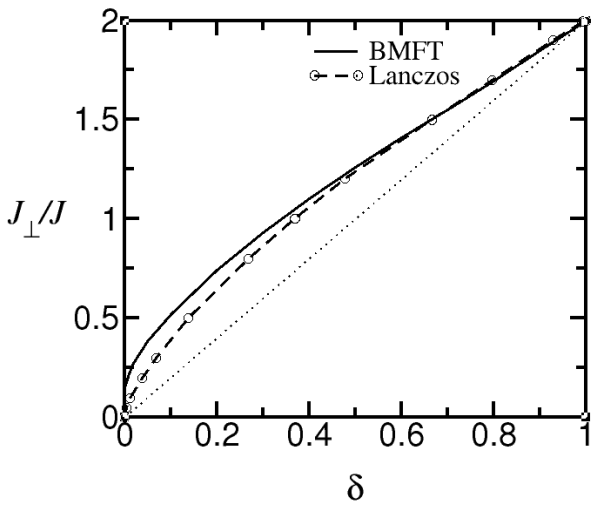

FIG. 5: Two-leg ladder: Critical line where the gap of the staggered phase vanishes. Along with the present bond meanfield theory predictions, the numerical diagonalization data from Ref. 9 are shown.

the uniform term $t$ and the dimerization susceptibility $\eta$ at $T=0$ are given in terms of the elliptic functions and can be found, e.g., in Ref. 24. In particular,

$$
\begin{aligned}
& \delta=0: Q_{ \pm}=\frac{1}{\pi} \\
& \delta=1: \quad Q_{+}=\frac{1}{2}, \quad Q_{-}=0 .
\end{aligned}
$$

For instance, at $\delta=1\left(J_{\perp}=0\right)$, the ladder reduces to the set of decoupled dimers with the Heisenberg exchange $2 J$. Our Eqs. (20,26) result in the exact value of the gap $\Delta=2 J$ for this case. Similar, at the integrable quantum critical point $\delta=1, J_{\perp}=2 J$ (uniform snakelike chain), we have $Q_{+}=P$, and the mean-field (23) correctly predicts a vanishing gap.

We plot in Fig. 5 the mean-field critical line given by

$$
\frac{J_{\perp}}{J}=\frac{2 u}{p} \delta
$$

along with the numerical results of Ref. 9. As one can see, the mean field works quite well quantitatively. It is surprising, but the mean field does much better than, e.g., the non-linear sigma model $(\mathrm{NL} \sigma \mathrm{M})$. The latter fails to predict the correct critical line. $\underline{4}$ Analysis of the topological term of the $\mathrm{NL} \sigma \mathrm{M}$ predicts that the line ends at $\delta=\frac{1}{2}, J_{\perp}=0$. The dotted line in Fig. 5 corresponds to the case when in the gap equations we set the bond parameters to their maximal values $Q_{ \pm}=P=\frac{1}{2}$ (i.e., $u=p=2$ ), so $J_{\perp} / J=2 \delta$. Such a naive "uniform isotropic limit" simplifies drastically the formulas, and provides a decent approximation. 


\section{THREE-LEG LADDER}

A three-leg dimerized ladder is defined by the Hamiltonian:

$$
\begin{aligned}
H_{3 \mathrm{~L}} & =\sum_{\alpha=1,2} \sum_{n=1}^{N} J_{\alpha}(n) \mathbf{S}_{\alpha}(n) \cdot \mathbf{S}_{\alpha}(n+1) \\
& +J_{\perp} \sum_{n=1}^{N}\left[\mathbf{S}_{1}(n) \cdot \mathbf{S}_{2}(n)+\mathbf{S}_{2}(n) \cdot \mathbf{S}_{3}(n)\right]
\end{aligned}
$$

Similar to the two-leg case, we consider dimerizations of the whole ladder (23) which follow the staggered or columnar patterns, as shown in Fig. 6.

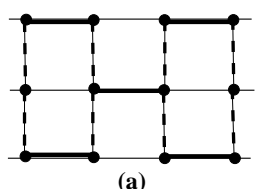

(a)

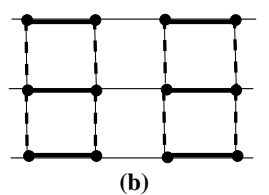

(b)
FIG. 6: Dimerized three-leg ladder. Line notations are the same as in Fig. 1 . Dimerization patterns: (a) - staggered; (b)- columnar.

Contrary to the previous case, now we don't have limits where the behavior of this ladder is known exactly. Although the non-dimerized limit $\delta=0$ is known to be gapless, and the three-leg ladder, roughly speaking, can be described as a "renormalized" spin- $\frac{1}{2}$ Heisenberg chain $\stackrel{1,16}{ }$ One can easily see from Fig. 6 that in the totally dimerized limit $\delta=1$ the columnar dimerized ladder becomes a set of $N / 2$ decoupled 6 -spin plaquettes, while the staggered pattern evolves into a "decorated" chain. The latter, to the best of our knowledge, has been not analyzed earlier.

We treat the problem in the bond mean-field approach as above. In fact, we will use directly our earlier results for the three-leg ladder coupled to phonons ${ }^{13}$, with minor modifications. Along with one couple of the bond parameters $Q_{ \pm}$, defined by (12) with $\alpha=1,3$, one needs an extra couple of parameters $Q_{ \pm}^{\prime}$ for the chain in the middle, defined by the same equation with $\alpha=2$. The effective Hamiltonian density $\mathcal{H}^{\mathrm{s} / \mathrm{c}}$ (cf. Eq. (13)) is now a $6 \times 6$ matrix, and the Nambu spinor $\Psi_{k}^{\dagger}=$ $\left(\begin{array}{cccccc}c_{1 k}^{A \dagger} & c_{1 k}^{B \dagger} & c_{2 k}^{A \dagger} & c_{2 k}^{B \dagger} & c_{3 k}^{A \dagger} & c_{3 k}^{B \dagger}\end{array}\right)$. The constant term

$$
\begin{aligned}
C_{3} & =J_{+}\left|Q_{+}\right|^{2}+J_{-}\left|Q_{-}\right|^{2}+\frac{1}{2} J_{-}\left|Q_{-}^{\prime}\right|^{2} \\
& +\frac{1}{2} J_{+}\left|Q_{+}^{\prime}\right|^{2}+2 J_{\perp}|P|^{2}
\end{aligned}
$$

Diagonalization of $\mathcal{H}^{\mathrm{s} / \mathrm{c}}$ results in six energy eigenvalues $\pm E_{j}^{\mathrm{s} / \mathrm{c}}(k), j=1,2,3$ for each configuration:

$$
E_{1}^{\mathrm{s}}(k)=E_{1}^{\mathrm{c}}(k)=\frac{1}{2} \sqrt{W}
$$

and

$$
E_{n}^{\mathrm{s} / \mathrm{c}}(k)=\frac{1}{2^{\frac{3}{2}}}\left[(-1)^{n} \sqrt{\left(W-W^{\prime}\right)^{2}+8 J_{\perp 1}^{2}\left(W+W^{\prime}+2 Y^{\mathrm{s} / \mathrm{c}}\right)}+W+W^{\prime}+4 J_{\perp 1}^{2}\right]^{\frac{1}{2}}, \quad n=2,3
$$

where

$Y^{\mathrm{s}}=\left(J_{1+} J_{1+}^{\prime}+J_{1-} J_{1-}^{\prime}\right) \cos (2 k)-\left(J_{1+} J_{1-}^{\prime}+J_{1-} J_{1+}^{\prime}\right)$, $Y^{\mathrm{c}}=\left(J_{1+} J_{1-}^{\prime}+J_{1-} J_{1+}^{\prime}\right) \cos (2 k)-\left(J_{1+} J_{1+}^{\prime}+J_{1-} J_{1-}^{\prime}\right)$,

and $J_{1 \pm}^{\prime}$ and $W^{\prime}$ are given by Eqs. (1617) where $Q_{ \pm} \rightarrow$ $Q_{ \pm}^{\prime}$. The mean-field equations are obtained via minimization of the free energy, given by Eq. (19) with $m=3$. Our analysis here follows the steps of the above two-leg case, so we will be brief.

Similar to the two-leg case, the columnar order corresponds to a state with lower energy of the dimerized three-leg ladder, thus this is a thermodynamically stable state. We present several plots of the zero-temperature energies for the two types of dimerization order in Fig. 7. The columnar phase of the three-leg ladder is always gapped when $\delta \neq 0$. The gap $\Delta_{c}=E_{1}^{\mathrm{c}}(0)$ is given by

$$
\Delta_{c}=J u \delta
$$

In the limit $\delta \rightarrow 0$, this mean-field approach correctly predicts gaplessness 16 , in accordance with the general results for odd- $m$-leg ladders $\frac{1,2}{}$ We should however point out that the gapless limit $\Delta_{c} \rightarrow 0$ when $\delta \rightarrow 0$ is recovered only if we set $\left(Q_{-}^{\sharp}-Q_{+}^{\sharp}\right) \rightarrow 0$. The latter limit, obviously correct from physical point of view [cf. Eq.(24)], is not automatically fulfilled in the bond meanfield equations. This is an artefact of the particular bond mean-field decoupling scheme, and is not intrinsic for any mean-field decoupling, as one see, e.g., from the (classical) mean-field results of Ref. 25. It is worth noting that the simple analytical approximations we derive, are free of these flaws.

The staggered phase is gapped everywhere, except for a critical line of vanishing gap, when $\delta \neq 0, J_{\perp} \neq 0$. Thus, we confirm the original conjecture ${ }^{4}$, which has been corroborated by the recent DMRG calculations of Almeida et $a l^{11}$. The gap of the staggered phase, found from 


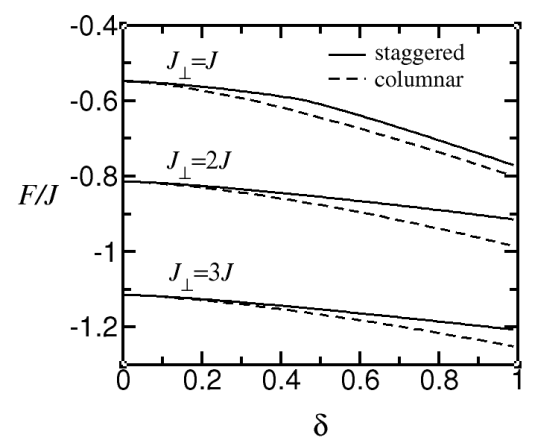

$E_{3}^{\mathrm{s}}(0)$, reads as

FIG. 7: Three-leg ladder: Energies of staggered and columnar dimerization configurations at $T=0$.

$$
\Delta_{s}^{<}=\frac{J}{\sqrt{2}}\left[\delta^{2}\left(u^{2}+u^{\prime 2}\right)+\left(\frac{J_{\perp} p}{J}\right)^{2}-\left(u+u^{\prime}\right) \sqrt{\left(u-u^{\prime}\right)^{2} \delta^{2}+2\left(\frac{J_{\perp} p}{J}\right)^{2}}\right]^{\frac{1}{2}}
$$

where $u^{\prime}$ is given by Eq. (21) with replacement $Q_{ \pm} \rightarrow$ $Q_{ \pm}^{\prime}$. The columnar gap $\Delta_{c}$ and the (critical) staggered gap $\Delta_{s}$, directly calculated from the mean-field equations, are plotted in Fig. 8 . There is one subtlety related

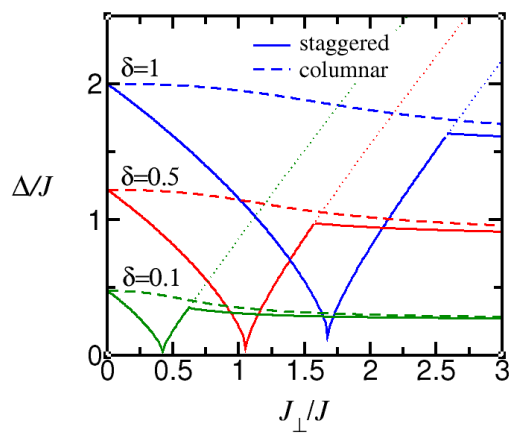

FIG. 8: Three-leg ladder: Gaps of the staggered and columnar phases.

to the staggered gap, absent in case of the two-leg ladder. Recall that we defined gap in a given phase as a minimal band gap of the energy eigenvalues of the effective single-particle Hamiltonian. What happens here is that the staggered gap (33) is determined from the eigenvalue $E_{3}^{\mathrm{s}}$ which has minimal band gap at small $J_{\perp} / J$. However with the increase of $J_{\perp} / J$ it reaches a certain value where "a level crossing" occurs, i.e., the band gaps of $E_{3}^{\mathbf{s}}$ and $E_{1}^{\mathrm{s}}$ are equal. Then, for bigger $J_{\perp} / J$, the staggered gap is determined by the (minimal) band gap of $E_{1}^{\mathrm{s}}$. In addition, since $E_{1}^{\mathrm{s}}=E_{1}^{\mathrm{c}}$ (30), the staggered and columnar gaps are equal, and according to (32), $\Delta_{s}^{>}=\Delta_{c}=J u \delta$ in that region. In fact, however, one can see differences between $\Delta_{s}^{>}$and $\Delta_{c}$. The reason is that the self-consistently determined mean-field parameters entering Eq. (21) for $u$, are not necessarily equal for two configurations.

To get some analytically tractable formulas for the gap, let us approximate $u=u^{\prime}$. Then

$$
\Delta_{s}^{<} \approx J\left|u \delta-\frac{p}{\sqrt{2}} \frac{J_{\perp}}{J}\right|
$$

Making even a more drastic approximation for the bond mean-field parameters by their maximal values, i.e., $u=$ 2 and $p=1+1 / \sqrt{2}\left(P=2^{-\frac{3}{2}}\right)$, we get

$$
\Delta_{s}^{<} \approx 2 J\left|\delta-\frac{1+\sqrt{2}}{4} \frac{J_{\perp}}{J}\right| .
$$

We plot in Fig. 9 the critical line obtained from the "exact" mean-field gap equation (33) and the DMRG result of Ref. 11. The dotted line $J_{\perp} / J=1.6569 \delta$ is the prediction of the simplified gap equation (35). Note that

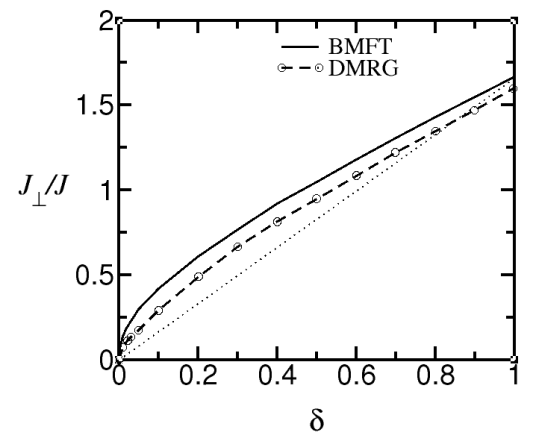

FIG. 9: Three-leg ladder: Critical line of the vanishing staggered gap. Our results are shown along with the DMRG data from Ref. 11.

contrary to the two-leg ladder, where we have exact predictions for the two integrable end points of the critical 
line, for this case we don't have an independent prediction for the critical ratio $J_{\perp} / J$ at $\delta=1.26$ We can point out however a very good agreement between the mean field and DMRG. The former via Eq. (33) yields 1.67 [or 1.66 via [35] $]$, while the latter yields 1.60 .

\section{CONCLUSIONS}

We analyze the possibility of quantum criticality (gaplessness) in dimerized two- and three-leg ladders. Contrary to earlier studies of these models, we do not imply a particular ladder's dimerization pattern. In this work we restrict ourselves to the zero-temperature properties. We find that for a given intrinsic intrachain dimerization, the ladder has lower zero-temperature energy for the columnar dimerization order. This is true for both types of ladders (two-, three-leg) considered. For the columnar dimerization pattern the ladders are always gapped, i.e., there are no lines of quantum criticality on the $\left(\delta, J_{\perp}\right)$ plane.

For the staggered dimerization order, we find that the ladders possess the quantum critical lines, in agreement with earlier analyses of this problem $5,6,7,8,9,10,11$ The mean-field theory we apply in this study demonstrates its quantitative accuracy. For the two-leg ladder, the meanfield critical line passes through both integrable quantum critical points $(0,0)$ and $(1,2 J)$ on the $\left(\delta, J_{\perp}\right)$ plane, and demonstrates a good agreement with the numerical diagonalization results 9 in between. For the case of three-leg ladder, the mean-field critical line passes through $(0,0)$ (the only integrable quantum critical point) and agrees well with the critical line determined through the DMRG calculations. 11

Thus, from a more practical point of view, the very possibility of quantum criticality in dimerized ladders hinges on some mechanism which would lock the dimerization order into a more energetically expensive staggered configuration. A realistic example of dimerization in a three-leg ladder through the spin-Peierls mechanism results in the columnar order and so, the gapful phase everywhere at non-zero dimerization. 13 At the moment we are not aware of any Hamiltonian with would provide such a mechanism for quantum criticality in dimerized ladders to occur.

\section{Acknowledgments}

We thank J. Almeida for useful correspondence. We acknowledge financial support from the Natural Science and Engineering Research Council of Canada (NSERC) and the Laurentian University Research Fund (LURF).
* Also at School of Science and Engineering, Al Akhawayn University, Ifrane 53000, Morocco.

1 E. Dagotto and T.M. Rice, Science 271, 618 (1996).

2 T. Giamarchi, Quantum Physics in One Dimension (Oxford University Press, Oxford, 2004).

3 P.A. Lee, N. Nagaosa, and X.-G. Wen, Rev. Mod. Phys. 78, 17 (2006).

4 M.A. Martin-Delgado, R. Shankar, and G. Sierra, Phys. Rev. Lett. 77, 3443 (1996).

5 M.A. Martin-Delgado, J. Dukelsky, and G. Sierra, Phys. Lett. A 250, 430 (1998).

6 V.N. Kotov, J. Oitmaa, and Z. Weihong, Phys. Rev. B 59, 11377 (1999).

7 D.C. Cabra and M.D. Grynberg, Phys. Rev. Lett. 82, 1768 (1999).

8 Y.-J. Wang and A.A. Nersesyan, Nucl. Phys. B 583 [FS], $671(2000)$.

9 K. Okamoto, Phys. Rev. B 67, 212408 (2003).

10 J. Almeida, M.A. Martin-Delgado, and G. Sierra, arXiv:0704.2181 (2007).

11 J. Almeida, M.A. Martin-Delgado, and G. Sierra, arXiv:0707.4452 (2007).

12 In this work we study the case of the antiferromagnetic (positive) spin exchange couplings only.

13 M. Azzouz, K. Shahin, and G.Y. Chitov, arXiv:0708.4181 (2007); K. Shahin, M. Sc. thesis, Laurentian University (2007).

14 M. Azzouz, Phys. Rev. B 48, 6136 (1993).

15 M. Azzouz, L. Chen, and S. Moukouri, Phys. Rev. B 50,
6233 (1994).

16 M. Azzouz and K. A. Asante, Phys. Rev. B 72, 094433 (2005).

17 See, e.g., E. Chattopadhyay and I. Bose, Physica A 318, 14 (2003).

18 J.L. Black and V.J. Emery, Phys. Rev. B 23, 429 (1981).

19 I. Affleck, D. Gepner, H.J. Schulz, and T. Ziman, J. Phys. A 22, 511 (1989);

20 T. Papenbrock, T. Barnes, D.J. Dean, M.V. Stoitsov, and M.R. Strayer, Phys. Rev. B 68, 024416 (2003).

21 E. Orignac, Eur. Phys. J. B 39, 335 (2004); E. Orignac and R. Chitra, Phys. Rev. B 70, 214436 (2004).

22 Note that contrary to the sine-Gordon formula (11), the logarithmically corrected dimerized chain energy (10) is lower than the plaquette energy (9) in the region $\delta_{\text {eff }} \gtrsim$ 0.4 . However, the formulas (10]9) are reliable only when $\delta_{\text {eff }} \ll 1$.

23 The other band $E_{2}^{\mathrm{s}}$ can be excluded from further considerations, since it has a bigger non-vanishing gap.

24 G.Y. Chitov and C. Gros, Phys. Rev. B 69, 104423 (2004).

25 L.N. Bulaevskii, Zh. Eksp. Teor. Fiz. 44, 1008 (1963) [Sov. Phys. JETP 17, 684 (1963)].

${ }^{26}$ Using the formula for the NL $\sigma \mathrm{M}$ topological term derived in Ref. 4, we obtain the critical line located between two points: $\left(\delta=\frac{18}{35}=0.5142 \ldots, J_{\perp}=0\right)$ and $\left(\delta=1, J_{\perp} / J=\right.$ $1.9823 \ldots)$. So, this approach does not result in adequate predictions. 